\title{
Orbital Stability of the Periodic Solutions of Autonomous Systems with Impulse Effect
}

\author{
By \\ Pavel S. Simeonov* and Drumi D. BaInov*
}

\begin{abstract}
In the present paper the orbital asymptotic stability of the periodic solutions of autonomous systems with impulse effect is investigated. An analogue of the theorem of Andronov-Vitt is proved.
\end{abstract}

\section{$\S 1 . \quad$ Introduction}

In the recent years still more works have been published dedicated to systems with impulse effect [1]-[9].

These systems describe evolution processes which in certain moments change rapidly their state. In the mathematical simulation of such processes it is convenient to neglect the duration of this rapid change and to assume that the system changes its state by jumps.

Processes with such a character are studied in numerous fields of science and techniques such as control theory, impulse techniques, populational dynamics, mass service, control of the reserves, etc.

Systems with impulse effect are defined by a system of ordinary differential equations $\dot{x}=f(t, x)$ and conditions which determine the moments and the magnitude of the impulse effect. The determination of the moments of impulse effect can be realized in various ways.

For instance, the moments of impulse effect for the system

$$
\begin{aligned}
& \frac{d x}{d t}=f(t, x), \quad t \neq \tau_{k}(x), \\
& \left.\Delta x\right|_{t=\tau_{k}(x)}=I_{k}(x)
\end{aligned}
$$

occur when the mapping point $(t, x(t))$ of the extended phase space meets some of the hypersurfaces $\sigma_{k}$ defined by the equations $t=\tau_{k}(x), k=1,2, \ldots$.

The moments of impulse effect of the system

Communicated by S. Hitotumatu, December 14, 1987.

* University of Plovdiv, Paisii Hilendarski, Plovdiv, Bulgaria.

This work was supported in part by the Committee for Science of the Council of Ministers of People's Republic of Bulgaria under Contract No 61. 


$$
\begin{aligned}
& \frac{d x}{d t}=f(t, x), t \neq \tau_{k}, \\
& \left.\Delta x\right|_{t=\tau_{k}}=I_{k}(x), k=1,2, \ldots
\end{aligned}
$$

are $t=\tau_{k}, k=1,2, \ldots$ and are fixed.

The moments of impulse effect of the system

$$
\begin{aligned}
& \frac{d x}{d t}=g(x), x \bar{\in} M, \\
& \left.\Delta x\right|_{x \in M}=I(x)
\end{aligned}
$$

occur when the point $x(t)$ of the phase space $X$ meets the set $M \subset X$.

The solutions $x(t)$ of systems $(\mathrm{a}, \mathrm{b}, \mathrm{c})$ are piecewise continuous functions. At the moment $t=\tau_{k}$ of impulse effect the solution $x(t)$ has a discontinuity of first type and we assume that the following equalities hold

$$
x\left(\tau_{k}-0\right)=x\left(\tau_{k}\right), x\left(\tau_{k}+0\right)=x\left(\tau_{k}\right)+\Delta x\left(\tau_{k}\right) .
$$

Between two consecutive moments of impulse effect $\left(t \in\left(\tau_{k}, \tau_{k+1}\right]\right)$ the solution $x(t)$ of system (a) coincides with the solution $\xi(t)$ of the initial value problem

$$
\frac{d \xi}{d t}=f(t, \xi), \xi\left(\tau_{k}\right)=x\left(\tau_{k}+0\right)
$$

The solutions of systems (b) and (c) for $t \in\left(\tau_{k}, \tau_{k+1}\right]$ are defined analogously.

We shall note that the moments of impulse effect of the different solutions of systems (a) and (c) are different. That is why in the investigation of such systems there are some additional difficulties which do not occur when we study system (b).

Up to the present moment systems of type (a) and (b) have been profoundly studied [1]-[9] while systems of type (c) have been almost not studied.

Note that in the case when $f(t, x)$ does not depend on $t$ (i.e. $f(t, x)=g(x)$ ), systems (a) and (b) do not possess the property of autonomy. System (c) has this property. On the other hand, in practice the most frequently one meets autonomous systems with impulse effect. That is why the investigation of systems of type (c) represents an undoubted interest.

In the present paper the orbital asymptotical stability of a periodic solution of system (c) is investigated and an analogue of the theorem of Andronov-Vitt is proved [10], [11].

\section{§2. Preliminary Notes}

Consider the autonomous system with impulse effect 


$$
\begin{aligned}
& \frac{d x}{d t}=g(x), x \bar{\in} M, \\
& \left.\Delta x\right|_{x \in M}=I(x),
\end{aligned}
$$

where $t \in \mathbb{R} ; g, I: \Omega \rightarrow \mathbb{R}^{n} ; \Omega$ is a domain contained in the $n$-dimensional Euclidean space $\mathbf{R}^{n}$ with elements $x=\operatorname{col}\left(x_{1}, \ldots, x_{n}\right)$, scalar product $(x, y)=x_{1} y_{1}$ $+\cdots+x_{n} y_{n}$ and norm $|x|=(x, x)^{1 / 2} ; M$ is an $(n-1)$-dimensional manifold contained in $\Omega$.

Further on we shall use the following notations: $|A|=\sup _{|x|=1}|A x|$-norm of the

$(n \times n)$-matrix $A$; $\operatorname{diag}\left(A_{1}, A_{2}\right)$ - a block-diagonal matrix with blocks $A_{1}$ and $A_{2} ; \quad E_{m}$-the unit $(m \times m)$-matrix; $0_{m}$-the zero $(m \times m)$-matrix; $B_{\varepsilon}\left(x_{0}\right)=$ $\left\{x \in \mathbf{R}^{n}:\left|x-x_{0}\right|<\varepsilon\right\}-\varepsilon$-neighbourhood of the point $x_{0} \in \mathbf{R}^{n} ; \bar{G}$-the closure of the set $G \subset \mathbb{R}^{n} ; \rho(x, L)=\inf _{y \in L}|x-y|$-the distance from the point $x \in \mathbb{R}^{n}$ to the set $L \subset \mathbf{R}^{n} ; \frac{\partial f}{\partial x}=\left(\frac{\partial f_{i}}{\partial x_{j}}\right)_{1}^{n}$ - the Jacobian matrix of the function $f: \mathbf{R}^{n} \rightarrow \mathbf{R}^{n}$; $[a ; b]$ - the interval $[a, b]$ if $a \leq b$ or the interval $[b, a]$ if $b<a ; x\left(t ; t_{0}, x_{0}\right)$ - the solution of system (1) satisfying the initial condition $x\left(t_{0}+0 ; t_{0}, x_{0}\right)=x_{0}$ and $J^{+}\left(t_{0}, x_{0}\right)$ - the maximal interval of the form $\left(t_{0}, \omega\right)$ in which the solution $x\left(t ; t_{0}, x_{0}\right)$ is continuable to the right.

Let $\varphi(t) \quad\left(t \in \mathbb{R}_{+}=[0, \infty)\right)$ be a solution of system (1) with moments of impulse effect $\left\{\tau_{k}\right\}$ :

$$
\begin{aligned}
& 0<\tau_{1}<\tau_{2}<\cdots, \lim _{k \rightarrow \infty} \tau_{k}=\infty \quad \text { and } \\
& L=\left\{x \in \mathbb{R}^{n}: x=\varphi(t), t \in \mathbb{R}_{+}\right\} .
\end{aligned}
$$

Definition 1. The solution $\varphi(t)$ of system (1) is called:

1.1. orbitally stable if

$$
\begin{aligned}
& (\forall \varepsilon>0)(\forall \eta>0)\left(\forall t_{0} \in \mathbf{R}_{+},\left|t_{0}-\tau_{k}\right|>\eta\right)(\exists \delta>0) \\
& \left(\forall x_{0} \in \Omega, \rho\left(x_{0}, L\right)<\delta, x_{0} \bar{\in} \bar{B}_{\eta}\left(\varphi\left(\tau_{k}\right)\right) \cup \bar{B}_{\eta}\left(\varphi\left(\tau_{k}+0\right)\right)\right)\left(\forall t \in J^{+}\left(t_{0}, x_{0}\right)\right) \\
& \rho\left(x\left(t ; t_{0}, x_{0}\right), L\right)<\varepsilon ;
\end{aligned}
$$

1.2. orbitally attractive if

$$
\begin{aligned}
& (\forall \eta>0)\left(\forall t_{0} \in R_{+},\left|t_{0}-\tau_{k}\right|>\eta\right)(\exists \lambda>0) \\
& \left(\forall x_{0} \in \Omega, \rho\left(x_{0}, L\right)<\lambda, x_{0} \bar{\in} \bar{B}_{\eta}\left(\varphi\left(\tau_{k}\right)\right) \cup \bar{B}_{\eta}\left(\varphi\left(\tau_{k}+0\right)\right)\right)(\forall \varepsilon>0)(\exists \sigma>0) \\
& t_{0}+\sigma \in J^{+}\left(t_{0}, x_{0}\right)\left(\forall t \geq t_{0}+\sigma, t \in J^{+}\left(t_{0}, x_{0}\right)\right) \\
& \rho\left(x\left(t ; t_{0}, x_{0}\right), L\right)<\varepsilon
\end{aligned}
$$


1.3. orbitally asymptotically stable if it is orbitally stable and orbitally attractive.

Definition 2. We shall say that the solution $\varphi(t)$ of system (1) has the property asymptotic phase if

$$
\begin{aligned}
& (\forall \eta>0)\left(\forall t_{0} \in \mathbb{R}_{+},\left|t_{0}-\tau_{k}\right|>\eta\right)(\exists \lambda>0) \\
& \left(\forall x_{0} \in \Omega,\left|x_{0}-\varphi\left(t_{0}\right)\right|<\lambda\right)(\exists c \in \mathbb{R})(\forall \varepsilon>0)(\exists \sigma>|c|) \\
& t_{0}+\sigma \in J^{+}\left(t_{0}, x_{0}\right)\left(\forall t \geq t_{0}+\sigma, t \in J^{+}\left(t_{0}, x_{0}\right),\left|t-\tau_{k}\right|>\eta\right) \\
& \left|x\left(t+c ; t_{0}, x_{0}\right)-\varphi(t)\right|<\varepsilon .
\end{aligned}
$$

Remark 1. Let the functions $g(x)$ and $I(x)$ be differentiable, the manifold $M$ be smooth and the normal vectors $n_{k}$ to $M$ at the points $\varphi\left(\tau_{k}\right)$ be such that $\left(n_{k}\right.$, $\left.g\left(\varphi\left(\tau_{k}\right)\right)\right) \neq 0, k=1,2, \ldots$. Then a straightforward verification shows that the function $\xi(t)=\frac{d \varphi}{d t}(t)$ satisfies the following linear system with impulse effect at fixed moments of time

$$
\begin{aligned}
& \frac{d u}{d t}=\frac{\partial g}{\partial x}(\varphi(t)) u, t \neq \tau_{k}, \\
& \left.\Delta u\right|_{t=\tau_{k}}=N_{k} u, k=1,2, \ldots,
\end{aligned}
$$

where

$$
N_{k} u=\frac{\partial I}{\partial x}\left(\varphi\left(\tau_{k}\right)\right) u+\left[g\left(\varphi\left(\tau_{k}+0\right)\right)-g\left(\varphi\left(\tau_{k}\right)\right)-\frac{\partial I}{\partial x}\left(\varphi\left(\tau_{k}\right)\right) g\left(\varphi\left(\tau_{k}\right)\right)\right] \frac{\left(n_{k}, u\right)}{\left(n_{k}, g\left(\varphi\left(\tau_{k}\right)\right)\right)}
$$

System (2) is called system in variations (of system (1) with respect to the solution $\varphi(t))$.

We shall say that conditions (A) hold if the following conditions are satisfied:

A1. System (1) has a T-periodic solution $p(t)$ with moments of impulse effect $\tau_{k}\left(\tau_{k}<\tau_{k+1}, k=0, \pm 1, \pm 2, \ldots\right)$ and the positive integer $q$ be such that

$$
\begin{aligned}
& \tau_{0}<0<\tau_{1}<\cdots<\tau_{q}<T<\tau_{q+1}, \\
& \tau_{k+q}=\tau_{k}+T, \quad k=0, \pm 1, \pm 2, \ldots .
\end{aligned}
$$

A2. $\frac{d p}{d t}(t) \neq 0$ for $t \neq \tau_{k}$,

A3. There exists a constant $H>0$ such that:

A3.1. The function $g: \Omega \rightarrow \mathbb{R}^{n}$ is differentiable in the set $D=\bigcup_{k=1}^{q} D_{k}(H) \subset \Omega$ and is continuous in $\bar{D}$ where 


$$
\begin{aligned}
& D_{k}(H)=\left\{x \in \mathbf{R}^{n}:|x-y|<H, y \in \Gamma_{k}\right\} \\
& \Gamma_{k}=\left\{x \in \mathbf{R}^{n}: x=p(t), t \in\left(\tau_{k-1}, \tau_{k}\right]\right\} \cup\left\{p\left(\tau_{k-1}+0\right)\right\} .
\end{aligned}
$$

A3.2. The function $I: \Omega \rightarrow \mathbf{R}^{n}$ is differentiable in the set $\bigcup_{k=1}^{q} B_{H}\left(p\left(\tau_{k}\right)\right) \subset \Omega$.

A3.3. The set $M \cap B_{H}\left(p\left(\tau_{k}\right)\right), k=1, \ldots, q$ coincides with the set of solutions of the equation $\phi(x)=0$ where the function $\phi: B_{H}\left(p\left(\tau_{k}\right)\right) \rightarrow \mathbf{R}$ is differentiable in $B_{H}\left(p\left(\tau_{k}\right)\right)$.

A4. The following relations hold

$$
\begin{aligned}
& \phi\left(p\left(\tau_{k}\right)\right)=0, k=1, \ldots, q \\
& \frac{\partial \phi}{\partial x}\left(p\left(\tau_{k}\right)\right) g\left(p\left(\tau_{k}\right)\right) \neq 0, k=1, \ldots, q .
\end{aligned}
$$

A5. For any $h \in(0, H)$ there exists $\gamma>0$ such that $\rho(x, M) \geq \gamma$ for $k=1, \ldots, q$ and $x \in \Gamma_{k} \mid B_{h}\left(p\left(\tau_{k}\right)\right)$.

A6. $\rho_{1}=1$ and $\left|\rho_{k}\right|<1, k=2, \ldots, n$ where $\rho_{k}, k=1, \ldots, n$ are the multiplicators of the $T$-periodic linear system with fixed moments of impulse effect

$$
\begin{aligned}
& \frac{d y}{d t}=\frac{\partial g}{\partial x}(p(t)) y, t \neq \tau_{k} \\
& \left.\Delta y\right|_{t=\tau_{k}}=N_{k} y, k=0, \pm 1, \pm 2, \ldots
\end{aligned}
$$

and

$$
\begin{aligned}
N_{k} y & =\frac{\partial I}{\partial x}\left(p\left(\tau_{k}\right)\right) y \\
& +\left[g\left(p\left(\tau_{k}+0\right)\right)-g\left(p\left(\tau_{k}\right)\right)-\frac{\partial I}{\partial x}\left(p\left(\tau_{k}\right)\right) g\left(p\left(\tau_{k}\right)\right)\right] \frac{\frac{\partial \phi}{\partial x}\left(p\left(\tau_{k}\right)\right) y}{\partial \phi}\left(p\left(\tau_{k}\right)\right) g\left(p\left(\tau_{k}\right)\right)
\end{aligned}
$$

Remark 2. Let conditions A1-A4 hold. Then, by Remark 1, the function $y=\frac{d p}{d t}$ is a T-periodic solution of system (4). In view of condition A2 we conclude that the linear T-periodic system (4) has a multiplicator equal to one.

System (4) coincides with the system in variations of system (1) with respect to the solution $p(t)$.

Remark 3. Condition (3) means that the trajectories of system (1) are not tangent to the manifold $M$ in some neighbourhood of the point $p\left(\tau_{k}\right)$. 


\section{§. Main Results}

Before we go to the proof of the main theorem we shall prove some auxiliary assertions.

Denote by $z\left(t ; t_{0}, z_{0}\right)$ the solution of the initial value problem

$$
\frac{d z}{d t}=g(z), z\left(t_{0}\right)=z_{0} \in \mathbf{R}^{n}
$$

Lemma $\mathbb{1}$. Let conditions A1-A4 hold.

Then there exists a number $h \in(0, H)$ such that for any $k=1, \ldots, q$ there exists a unique differentiable function $T_{k}: B_{h}\left(p\left(\tau_{k}\right)\right) \rightarrow \mathbf{R}, w \rightarrow T_{k}(w)$ so that

$$
\begin{gathered}
T_{k}\left(p\left(\tau_{k}\right)\right)=\tau_{k}, \\
\phi\left(z\left(T_{k}(w) ; \tau_{k}, w\right)\right) \equiv 0, \\
\frac{\partial \phi}{\partial x}\left(z\left(T_{k}(w) ; \tau_{k}, w\right)\right)\left[g\left(z\left(T_{k}(w) ; \tau_{k}, w\right)\right) \frac{\partial T_{k}}{\partial w}(w)+\frac{\partial z}{\partial w}\left(T_{k}(w) ; \tau_{k}, w\right)\right] \equiv 0
\end{gathered}
$$

for $w \in B_{h}\left(p\left(\tau_{k}\right)\right)$.

Proof. Lemma 1 follows immediately from the implicit function theorem applied to the function

$$
\begin{gathered}
\varphi_{k}:\left(\tau_{k}-H, \tau_{k}+H\right) \times B_{H}\left(p\left(\tau_{k}\right)\right) \rightarrow \mathbb{R},(t, w) \rightarrow \varphi_{k}(t, w) \\
=\phi\left(z\left(t ; \tau_{k}, w\right)\right) \text { and the system } \varphi_{k}(t, w)=0 .
\end{gathered}
$$

Corollary 1. There exists a constant $\tau>0$ such that

$$
\left|T_{k}(w)-T_{k}(u)\right| \leq \tau|w-u| \text { for } k=1, \ldots, q \text { and } w, u \in B_{h}\left(p\left(\tau_{k}\right)\right) .
$$

Let $n>0$ and $m>0$ be integers and $D \subset R$. Denote by $P C\left(D, R^{n \times m}\right)$ the space of $(n \times m)$-matrix-valued functions which are defined for $t \in D$, continuous in $t \in D, t \neq \tau_{k}$, at the points $\tau_{k}$ they have discontinuities of first type and are left continuous.

The following two lemmas are related to the system

$$
\begin{aligned}
& \frac{d y}{d t}=P(t) y, t \neq \tau_{k}, \\
& \left.\Delta y\right|_{t=\tau_{k}}=P_{k} y, k=0, \pm 1, \pm 2, \ldots,
\end{aligned}
$$

where $P(t)$ and $P_{k}(t \in R, k=0, \pm 1, \pm 2, \ldots)$ are $(n \times n)$-matrices.

Introduce the following conditions $(C)$ :

C1. $P(\cdot) \in P C\left(R, R^{n \times n}\right)$ and $P(t+T)=P(t)$ for $t \in \mathbb{R}$.

C2. There exists an integer $q>0$ such that 


$$
\tau_{k+q}=\tau_{k}+T, P_{k+q}=P_{k}, k=0, \pm 1, \pm 2, \ldots .
$$

C3. $\rho_{1}=1,\left|\rho_{k}\right|<1, k=2, \ldots, n$,

where $\rho_{k}, k=1, \ldots, n$ are the multiplicators of system (6).

Lemma 2. Let conditions (C) hold.

Then system (6) has a fundamental matrix of the form

$$
Y(t)=\psi(t) \operatorname{diag}\left(E_{1}, e^{C t}\right),
$$

where the matrix $\psi(\cdot) \in P C\left(\mathbf{R}, \mathbf{R}^{n \times n}\right)$ is non-singular and $2 T$-periodic and $C$ is a real $(n-1) \times(n-1)$-matrix with eigenvalues which have negative real parts:

$$
\operatorname{Re} \lambda_{j}(C)<0, j=1, \ldots, n-1 .
$$

Proof. Let $\tilde{Y}(t)$ be the fundamental matrix of system (6) for which $\tilde{Y}(0)$ $=E_{n}$. From the $T$-periodicity of system (6) it follows that

$$
\tilde{Y}(t+T)=\tilde{Y}(t) \tilde{Y}(T) \text { for } t \in \mathbf{R} .
$$

From condition $\mathrm{C} 3$ it follows that the monodromy matrix $\tilde{Y}(T)$ has eigenvalues

$$
\rho_{1}=1 \text { and }\left|\rho_{k}\right|<1, \quad k=2, \ldots, n .
$$

Hence there exists a non-singular matrix $S$ such that

$$
S^{-1} \tilde{Y}(T) S=\operatorname{diag}\left(E_{1}, B_{1}\right)=B,
$$

where $B_{1}$ is a real $(n-1) \times(n-1)$-matrix with eigenvalues which are in modulus less than one:

$$
\left|\lambda_{j}\left(B_{1}\right)\right|<1, \quad j=1, \ldots, n-1 .
$$

Set

$$
\begin{aligned}
& C=\frac{1}{T} \ln B_{1}, \\
& \Lambda=\frac{1}{T} \ln B=\operatorname{diag}\left(0_{1}, C\right), \\
& Y(t)=\tilde{Y}(t) S .
\end{aligned}
$$

Since from (13), (9) and (10) it follows that

$$
Y(t+T)=\tilde{Y}(t+T) S=\tilde{Y}(t) \tilde{Y}(T) S=\tilde{Y}(t) S S^{-1} \tilde{Y}(T) S=Y(t) B
$$

then by the representation of Floquet the fundamental matrix $Y(t)$ has the form

$$
Y(t)=\psi(t) e^{\Lambda t}=\psi(t) \operatorname{diag}\left(E_{1}, e^{C t}\right),
$$

where the matrix $\psi(\cdot) \in P C\left(\mathbf{R}, \mathbf{R}^{n \times n}\right)$ is non-singular and $2 T$-periodic. 
From (11) and (12) there follows (8) which proves Lemma 2.

Lemma 3. Let conditions (C) hold, the fundamental matrix $Y(t)$ of system (6) have the form (7) and

$$
G(t, s)=\left\{\begin{array}{r}
Y(t) \operatorname{diag}\left(0_{1}, E_{n-1}\right) Y^{-1}(s), t>s \\
-Y(t) \operatorname{diag}\left(E_{1}, 0_{n-1}\right) Y^{-1}(s), t \leq s
\end{array}\right.
$$

Then:

1) The matrix $G(t, s)$ satisfies the relations

$$
\begin{aligned}
& G(t, t-0)-G(t, t+0)=E_{n}, \quad t \in \mathbb{R}, \\
& \frac{\partial G}{\partial t}(t, s)=P(t) G(t, s), \quad t \neq \tau_{k}, \\
& G\left(\tau_{k}+0, s\right)=\left(E_{n}+P_{k}\right) G\left(\tau_{k}, s\right), \tau_{k} \neq s, \\
& G\left(\tau_{k}+0, \tau_{k}\right)=\left(E_{n}+P_{k}\right) G\left(\tau_{k}, \tau_{k}\right)+E_{n}, k=0, \pm 1, \pm 2, \ldots, \\
& |G(t, s)| \leq \begin{cases}K e^{-2 \alpha(t-s)}, & t>s \\
K & t \leq s,\end{cases}
\end{aligned}
$$

where $\alpha>0$ and $K \geq 1$ are constants.

2) If $b_{k} \in \mathbb{R}^{n}, f \in P C\left(\mathbf{R}_{+}, \mathbb{R}^{n \times 1}\right), a \in \mathbb{R}^{n}, e_{1}=\operatorname{col}(1,0, \ldots, 0)$ and

$$
\begin{aligned}
& \int_{0}^{\infty}|f(t)| d t+\sum_{k=1}^{\infty}\left|b_{k}\right|<\infty, \\
& \left(a, e_{1}\right)=0
\end{aligned}
$$

then the function

$$
y(t, a)=Y(t) a+\int_{0}^{\infty} G(t, s) f(s) d s+\sum_{k=1}^{\infty} G\left(t, \tau_{k}\right) b_{k} \quad\left(t \in \mathbb{R}_{+}\right)
$$

is a solution of the system

$$
\begin{aligned}
& \frac{d y}{d t}=P(t) y+f(t), \quad t \neq \tau_{k}, \\
& \left.\Delta y\right|_{t=\tau_{k}}=P_{k} y+b_{k}, \quad k=1,2, \ldots
\end{aligned}
$$

and

$$
\lim _{t \rightarrow \infty} y(t, a)=0
$$

3) If, moreover,

$$
|f(t)| \leq r e^{-\alpha t},\left|b_{k}\right| \leq r e^{-\alpha \tau_{k}}, t \in \mathbb{R}_{+}, \tau_{k} \in \mathbb{R}_{+},
$$


then the function

$$
u(t)=\int_{0}^{\infty} G(t, s) f(s) d s+\sum_{k=1}^{\infty} G\left(t, \tau_{k}\right) b_{k}
$$

satisfies the estimate

$$
|u(t)| \leq Q r e^{-\alpha t}, t \in \mathbf{R}_{+},
$$

where the constant $Q>0$ is independent of $r, f(t)$ and $b_{k}$.

Proof. Equalities (15)-(18) follows immediately from formula (14).

From representation (7) and formula (14) it follows that

$$
G(t, s)=\left\{\begin{array}{l}
\psi(t) \operatorname{diag}\left(0_{1}, e^{C(t-s)}\right) \psi_{(s)}^{-1}, t>s \\
-\psi(t) \operatorname{diag}\left(E_{1}, 0_{n-1}\right) \psi^{-1}(s), t \leq s .
\end{array}\right.
$$

By Lemma 2 there exists a number $\alpha>0$ such that

$$
\operatorname{Re} \lambda_{j}(C)<-2 \alpha<0, j=1, \ldots, n-1
$$

and the matrices $\psi(t)$ and $\psi^{-1}(t)$ are bounded on $\mathbf{R}$ (since they are periodic and belong to $P C\left(\mathbf{R}, \mathbf{R}^{n \times n}\right)$ ). Then (26) and (27) imply estimate (19).

2) Since $|G(t, s)| \leq K$ for $t, s \geq 0$, then

$$
\int_{0}^{\infty}|G(t, s) f(s)| d s+\sum_{k=1}^{\infty}\left|G\left(t, \tau_{k}\right) b_{k}\right| \leq K \int_{0}^{\infty}|f(s)| d s+K \sum_{k=1}^{\infty}\left|b_{k}\right|<\infty,
$$

hence the improper integral and the series in (21) are absolutely convergent.

Write (21) in the form

$$
\begin{aligned}
y(t, a)= & Y(t) a+\int_{0}^{t} G(t, s) f(s) d s+\int_{t}^{\infty} G(t, s) f(s) d s \\
& +\sum_{k=1}^{\infty} G\left(t, \tau_{k}\right) b_{k} .
\end{aligned}
$$

After a differentiation with respect to $t \neq \tau_{k}$, in view of (15) and (16), we obtain

$$
\begin{aligned}
\frac{d y}{d t}(t, a)= & \frac{d Y}{d t}(t) a+[G(t, t-0)-G(t, t+0)] f(t) \\
& +\int_{0}^{\infty} \frac{\partial G}{\partial t}(t, s) f(s) d s+\sum_{k=1}^{\infty} \frac{\partial G}{\partial t}\left(t, \tau_{k}\right) b_{k} \\
= & P(t) y(t, a)+f(t) .
\end{aligned}
$$

The differentiation is possible since the improper integral and the sum obtained as a result of the formal differentiation are convergent uniformly with respect to $t$ belonging to any finite subinterval of $\mathbf{R}_{+}$. 
Applying (17) and (18), we find that

$$
\begin{aligned}
y\left(\tau_{i}+0, a\right)= & Y\left(\tau_{i}+0, a\right)+\int_{0}^{\infty} G\left(\tau_{i}+0, s\right) f(s) d s \\
& +\sum_{\substack{k \neq 1 \\
k \neq i}}^{\infty} G\left(\tau_{i}+0, \tau_{k}\right) b_{k}+G\left(\tau_{i}+0, \tau_{i}\right) b_{i} \\
= & \left(E_{n}+P_{i}\right) y\left(\tau_{i}, a\right)+b_{i} .
\end{aligned}
$$

Hence $y(t, a)$ is a solution of (22).

In view of the structure of $Y(t)$ and of (20), we obtain that for $t \in \mathbb{R}_{+}$

$$
|Y(t) a| \leq K|a| e^{-2 \alpha t}
$$

Moreover,

$$
\begin{aligned}
0 \leq & \left|\int_{0}^{\infty} G(t, s) f(s) d s+\sum_{k=1}^{\infty} G\left(t, \tau_{k}\right) b_{k}\right| \\
\leq & \int_{0}^{t / 2}|G(t, s)||f(s)| d s+\int_{t / 2}^{\infty}|G(t, s)||f(s)| d s \\
& +\sum_{0<\tau_{k}<t / 2}\left|G\left(t, \tau_{k}\right)\right|\left|b_{k}\right|+\sum_{\tau_{k} \geq t / 2}\left|G\left(t, \tau_{k}\right)\right|\left|b_{k}\right| \\
\leq & \int_{0}^{t / 2} K e^{-2 \alpha(t-s)}|f(s)| d s+\int_{t / 2}^{\infty} K|f(s)| d s \\
& +\sum_{0<\tau_{k}<t / 2} K e^{-2 \alpha\left(t-\tau_{k}\right)}\left|b_{k}\right|+\sum_{\tau_{k} \geq t / 2} K\left|b_{k}\right| \\
\leq & K e^{-\alpha t} \int_{0}^{\infty}|f(s)| d s+K \int_{t / 2}^{\infty}|f(s)| d s \\
& +K e^{-\alpha t} \sum_{k=1}^{\infty}\left|b_{k}\right|+K \sum_{\tau_{k} \geq t / 2}\left|b_{k}\right| \rightarrow 0 \text { as } t \rightarrow \infty .
\end{aligned}
$$

Hence from (21), (28) and (29) it follows that relation (23) is fulfilled.

3) Let $f(t)$ and $b_{k}$ satisfy estimates (24),

$$
\min _{k}\left(\tau_{k}-\tau_{k-1}\right)=\theta \quad \text { and } \tau_{n}<t \leq \tau_{n+1} .
$$

Then the estimates

$$
\begin{aligned}
|u(t)| \leq & \int_{0}^{t} K e^{-2 \alpha(t-s)} r e^{-\alpha s} d s+\int_{t}^{\infty} K r e^{-\alpha s} d s \\
& +\sum_{k=1}^{n} K e^{-2 \alpha\left(t-\tau_{k}\right)} r e^{-\alpha \tau_{k}}+\sum_{k=n+1}^{\infty} K r e^{-\alpha \tau_{k}}
\end{aligned}
$$




$$
\begin{aligned}
e^{-2 \alpha t} \int_{0}^{t} e^{\alpha s} d s & +\int_{t}^{\infty} e^{-\alpha s} d s<\frac{2}{\alpha} e^{-\alpha t} \\
e^{-2 \alpha t} \sum_{k=1}^{n} e^{\alpha \tau_{k}} & \leq e^{-2 \alpha t}\left[\frac{1}{\theta} \sum_{k=1}^{n-1}\left(\tau_{k+1}-\tau_{k}\right) e^{\alpha \tau_{k}}+e^{\alpha \tau_{n}}\right] \\
& \leq e^{-2 \alpha t}\left[\frac{1}{\theta} \int_{\tau_{1}}^{\tau_{n}} e^{\alpha s} d s+e^{\alpha \tau_{n}}\right] \\
& \leq e^{-2 \alpha t}\left[\frac{1}{\alpha \theta}\left(e^{\alpha \tau_{n}}-e^{\alpha \tau_{1}}\right)+e^{\alpha \tau_{n}}\right] \\
& <e^{-2 \alpha t}\left(\frac{1}{\alpha \theta}+1\right) e^{\alpha \tau_{n}} \leq \frac{1+\alpha \theta}{\alpha \theta} e^{-\alpha t} \\
\sum_{k=n+1}^{\infty} e^{-\alpha \tau_{k}} & \leq e^{-\alpha \tau_{n+1}}+\frac{1}{\theta} \sum_{k=n+2}^{\infty}\left(\tau_{k}-\tau_{k-1}\right) e^{-\alpha \tau_{k}} \\
& \leq e^{-\alpha \tau_{n+1}}+\frac{1}{\theta} \int_{\tau_{n+1}}^{\infty} e^{-\alpha s} d s \\
& =\frac{1+\alpha \theta}{\alpha \theta} e^{-\alpha \tau_{n+1}} \leq \frac{1+\alpha \theta}{\alpha \theta} e^{-\alpha t}
\end{aligned}
$$

imply estimate (25) with $Q=\frac{2 K}{\alpha \theta}(\alpha \theta+\theta+1)$.

This completes the proof of Lemma 3.

Theorem 1. Let conditions (A) hold.

Then the T-periodic solution $p(t)$ of system (1) is orbitally asymptotically stable and has the property asymptotic phase.

Proof. Let in system (1) the change of the variables be realized

$$
x=S z+p(0)
$$

where the non-singular matrix $S$ is chosen so that

$$
S^{-1} \frac{d p}{d t}(0)=e_{1}=\operatorname{col}(1,0, \ldots, 0)
$$

As a result of this change we obtain a new system which has a $T$-periodic solution $\pi(t)=S^{-1}(p(t)-p(0))$. An immediate verification shows that the new system and the solution $\pi(t)$ satisfy conditions (A). Moreover, $\pi(0)=0$, $\frac{d \pi}{d t}(0)=e_{1}$. That is why, without loss of generality, we assume that the solution $p(t)$ of system (1) satisfies the conditions

$$
p(0)=0, \quad \frac{d p}{d t}(0)=e_{1}
$$


For the system in variations (4) the conditions of Lemma 2 hold with $P(t)$ $=\frac{\partial g}{\partial x}(p(t))$ and $P_{k}=N_{k}$. Hence system (4) has a fundamental matrix of the form

$$
Y(t)=\psi(t) \operatorname{diag}\left(E_{1}, e^{C t}\right),
$$

where the matrix $\psi(\cdot) \in P C\left(\mathbb{R}, \mathbb{R}^{n \times n}\right)$ is $2 T$-periodic non-singular and $\operatorname{Re} \lambda_{j}(C)<0$, $j=1, \ldots, n-1$.

From (31) we obtain that the first column of $Y(t)$ is a $2 T$-periodic solution of system (4). But from conditions $\mathrm{A} 2$ and A6 it follows that this solution is proportional to the T-periodic solution $\frac{d p}{d t}(t)$ of system (4). Hence we can assume that

$$
Y(t)=\left[\frac{d p}{d t}(t), Y_{1}(t)\right]
$$

where $Y_{1}(t)$ is an $n \times(n-1)$-matrix. Moreover, (30) implies

$$
Y(0)=\left[e_{1}, Y_{1}(0)\right]
$$

Let

$$
G(t, s)=\left\{\begin{array}{l}
Y(t) \operatorname{diag}\left(0_{1}, E_{n-1}\right) Y^{-1}(s), t>s \\
-Y(t) \operatorname{diag}\left(E_{1}, O_{n-1}\right) Y^{-1}(s), t \leq s
\end{array}\right.
$$

By Lemma $3 G(t, s)$ satisfies estimate (19) and $Y(t)$ satisfies estimate (28) if $a \in \mathbb{R}^{n},\left(a, e_{1}\right)=0$.

Choose successively $h \in(0, H)$ by Lemma 1 and $\gamma>0$ by condition A5.

First we shall prove that there exist constants $\eta_{0}>0$ and $B>0$ such that for any $a \in D\left(\eta_{0}\right)=\left\{a \in R^{n}:\left(a, e_{1}\right)=0,|a|<\eta_{0}\right\}$ system (1) has a solution $x(t)$ $=x(t, a)$ which is defined for $t \in \mathbf{R}_{+}$, has points of discontinuity $t_{k}=t_{k}(a)$ and satisfies the estimates

$$
\begin{aligned}
& \left|t_{k}-\tau_{k}\right| \leq B|a| e^{-\alpha \tau_{k}}=\delta_{k}, \\
& |x(t)-p(t)| \leq B|a| e^{-\alpha t} \quad \text { for }\left|t-\tau_{k}\right|>\delta_{k} .
\end{aligned}
$$

For this purpose we construct the sequences

$$
w_{n}(t)=w_{n}(t, a) \text { and } t_{k}^{n}=t_{k}^{n}(a), n=0,1,2, \ldots
$$

setting for $t \in R$ and $k=1,2, \ldots$

$$
W_{0}(t)=p(t), t_{k}^{0}=\tau_{k}
$$

after which we successively define

$$
w_{n+1}(t)=p(t)+Y(t) a+\int_{0}^{\infty} G(t, s) f_{n}(s) d s+\sum_{k=1}^{\infty} G\left(t, \tau_{k}\right) b_{k}^{n},
$$


where

$$
\begin{aligned}
f_{n}(t)= & F\left(w_{n}(t), t\right)=g\left(w_{n}(t)\right)-g(p(t))-\frac{\partial g}{\partial x}(p(t))\left(w_{n}(t)-p(t)\right), \\
b_{k}^{n}= & \left.\beta_{k}\left(w_{n}\left(\tau_{k}\right)\right)=I\left(z\left(t_{k}^{n} ; \tau_{k}, w_{n} \tau_{k}\right)\right)\right)-I\left(p\left(\tau_{k}\right)\right) \\
& +\int_{t_{k}^{n}}^{\tau_{k}}\left[g\left(z\left(s ; \tau_{k}, w_{n}\left(\tau_{k}+0\right)\right)\right)-g\left(z\left(s ; \tau_{k}, w_{n}\left(\tau_{k}\right)\right)\right)\right] d s \\
& -N_{k}\left(w_{n}\left(\tau_{k}\right)-p\left(\tau_{k}\right)\right) ;
\end{aligned}
$$

$t_{k}^{n+1}$ we determine as the unique solution with respect to $t$ of the system

$$
\phi\left(z\left(t ; \tau_{k}, w_{n+1}\left(\tau_{k}\right)\right)\right)=0
$$

i. e. $t_{k}^{n+1}=T_{k}\left(w_{n+1}\left(\tau_{k}\right)\right)$.

Let $|a|<\frac{1}{2 K} \min (h, \gamma)$. Then for $n=0$ from (37), (38), (36) and (28) it follows that

$$
\left|w_{1}(t)-p(t)\right| \leq K|a| e^{-2 \alpha t}, t \geq 0 .
$$

Moreover, since $\left|w_{1}\left(\tau_{k}\right)-p\left(\tau_{k}\right)\right| \leq K|a|<h$, then by Lemma 1 and Corollary 1 there exists a unique solution $t_{k}^{1}=T_{k}\left(w_{1}\left(\tau_{k}\right)\right)$ of the system $\phi\left(z\left(t ; \tau_{k}, w_{1}\left(\tau_{k}\right)\right)\right)=0$ and the following estimate holds

$$
\begin{aligned}
\left|t_{k}^{1}-t_{k}^{0}\right| & =\left|t_{k}^{1}-\tau_{k}\right|=\left|T_{k}\left(w_{1}\left(\tau_{k}\right)\right)-T_{k}\left(p\left(\tau_{k}\right)\right)\right| \\
& \leq \tau\left|w_{1}\left(\tau_{k}\right)-p\left(\tau_{k}\right)\right| \leq \tau K|a| e^{-2 \alpha \tau_{k}} .
\end{aligned}
$$

Let $\mu \in(0, h)$. The analysis shows that the functions $F(w, t)$ and $\beta_{k}(w)$ satisfy the inequalities

$$
|F(w, t)-F(u, t)| \leq L(\mu)|w-u| \text { for }|w-p(t)| \leq \mu,|u-p(t)| \leq \mu
$$

and

$$
\left|\beta_{k}(w)-\beta_{k}(u)\right| \leq L(\mu)|w-u|
$$

for $\left|w-p\left(\tau_{k}\right)\right| \leq \mu,\left|u-p\left(\tau_{k}\right)\right| \leq \mu$ where $\lim _{\mu \rightarrow 0+} L(\mu)=0$.

Choose $\mu \in\left(0, \frac{h}{K}\right)$ and $\eta_{0}>0$ so that

$$
L(\mu) Q=L(\mu) \frac{2 K}{\alpha \theta}(\alpha \theta+\theta+1)<\frac{1}{2}, \quad 2 K \eta_{0}<\mu .
$$

Then by induction with respect to $n$ we prove that the members of the sequences $w_{n}(t), t_{k}^{n}$ can be determined successively and the following estimates hold 


$$
\begin{aligned}
& \left|w_{n}(t)-w_{n-1}(t)\right| \leq K|a| 2^{1-n} e^{-\alpha t}, \\
& \left|w_{n}(t)-p(t)\right| \leq 2 K|a| e^{-\alpha t}, \\
& \left|t_{k}^{n}-t_{k}^{n-1}\right| \leq \tau K|a| 2^{1-n} e^{-\alpha \tau_{k}}, \\
& \left|t_{k}^{n}-\tau_{k}\right| \leq 2 \tau K e^{-\alpha \tau_{k}}
\end{aligned}
$$

for $n=1,2, \ldots, m$.

In fact, from (40) and (41) it follows that estimates (45)-(48) hold for $n$ $=1$. Let these estimates hold for $n=1, \ldots, m$. Then $\left|w_{k}(t)-p(t)\right|<\mu, k$ $=1, \ldots, m$ and from (42), (43) and (45) we find

$$
\begin{aligned}
\left|f_{m}(t)-f_{m-1}(t)\right| & =\left|F\left(w_{m}(t), t\right)-F\left(w_{m-1}(t), t\right)\right| \\
& \leq L(\mu)\left|w_{m}(t)-w_{m-1}(t)\right| \leq L(\mu) K|a| 2^{1-m} e^{-\alpha t}
\end{aligned}
$$

and

$$
\left|b_{k}^{m}-b_{k}^{m-1}\right| \leq L(\mu) K|a| 2^{1-m} e^{-\alpha \tau_{k}} .
$$

After this, by assertion 3 of Lemma 3, in view of (36) and (44), we obtain

$$
\begin{aligned}
\left|w_{m+1}(t)-w_{m}(t)\right| & =\left|\int_{0}^{\infty} G(t, s)\left[f_{m}(s)-f_{m-1}(s)\right] d s+\sum_{k=1}^{\infty} G\left(t, \tau_{k}\right)\left[b_{k}^{m}-b_{k}^{m-1}\right]\right| \\
& \leq Q L(\mu) K|a| 2^{1-m} e^{-\alpha t} \leq K|a| 2^{-m} e^{-\alpha t}
\end{aligned}
$$

From (49) it follows immediately that

$$
\begin{aligned}
& \left|w_{m+1}(t)-w_{m}(t)\right| \leq \sum_{j=1}^{m+1}\left|w_{j}(t)-w_{j-1}(t)\right| \\
& \leq K|a|\left(1+2^{-1}+2^{-2}+\cdots\right) e^{-\alpha t}=2 K|a| e^{-\alpha t} .
\end{aligned}
$$

In particular, $\left|w_{m+1}\left(\tau_{k}\right)-p\left(\tau_{k}\right)\right|<2 K|a|<h$ and by Lemma 1 equation (39) has a unique solution $t_{k}^{m+1}=T_{k}\left(w_{m+1}\left(\tau_{k}\right)\right)$ for which by corollary 1 we have

$$
\begin{aligned}
\left|t_{k}^{m+1}-t_{k}^{m}\right| & =\left|T_{k}\left(w_{m+1}\left(\tau_{k}\right)\right)-T_{k}\left(w_{m}\left(\tau_{k}\right)\right)\right| \leq \tau\left|w_{m+1}\left(\tau_{k}\right)-w_{m}\left(\tau_{k}\right)\right| \\
& \leq \tau K|a| 2^{-m} e^{-\alpha \tau_{k}} .
\end{aligned}
$$

Then

$$
\left|t_{k}^{m+1}-\tau_{k}\right| \leq \sum_{j=1}^{m+1}\left|t_{k}^{j}-t_{k}^{j-1}\right| \leq 2 \tau K|a| e^{-\alpha \tau_{k}} .
$$

Thus estimates (45) (48) hold for $n=m+1$, hence for each $n=1,2, \ldots$.

From (45) and (47) it follows that the sequences $w_{n}(t)$ and $t_{k}^{n}$ are convergent uniformly on $t \in \mathbb{R}$ and $k=1,2, \ldots$ Let $w(t)=w(t, a)$ and $t_{k}=t_{k}(a)$ be their limits. Then 


$$
\begin{gathered}
|w(t)-p(t)| \leq 2 K|a| e^{-\alpha t} \\
\left|t_{k}-\tau_{k}\right| \leq 2 \tau K|a| e^{-\alpha \tau_{k}}
\end{gathered}
$$

and

$$
w(t)=p(t)+Y(t) a+\int_{0}^{\infty} G(t, s) f(s, a) d s+\sum_{k=1}^{\infty} G\left(t, \tau_{k}\right) b_{k}(a)
$$

where

$$
f(t, a)=g(w(t))-g(p(t))-\frac{\partial g}{\partial x}(p(t))(w(t)-p(t))
$$

and

$$
\begin{aligned}
b_{k}(a)= & I\left(z\left(t_{k} ; \tau_{k}, w\left(\tau_{k}\right)\right)\right)-I\left(p\left(\tau_{k}\right)\right) \\
& +\int_{t_{k}}^{\tau_{k}}\left[g\left(z\left(s ; \tau_{k}, w\left(\tau_{k}+0\right)\right)\right)-g\left(z\left(s ; \tau_{k}, w\left(\tau_{k}\right)\right)\right)\right] d s \\
& -N_{k}\left(w\left(\tau_{k}\right)-p\left(\tau_{k}\right)\right) .
\end{aligned}
$$

In view of assertion 2 of Lemma 3 we obtain that $w(t)$ is a solution of the system with impulse effect in fixed moments of time

$$
\begin{aligned}
& \frac{d w}{d t}=g(w), t \neq \tau_{k}, \\
& \begin{aligned}
\left.\Delta w\right|_{t=\tau_{k}}= & I\left(z\left(t_{k} ; \tau_{k}, w\left(\tau_{k}\right)\right)\right) \\
& \quad+\int_{t_{k}}^{\tau_{k}}\left[g\left(z\left(s ; \tau_{k}, w\left(\tau_{k}+0\right)\right)\right)-g\left(z\left(s ; \tau_{k}, w\left(\tau_{k}\right)\right)\right)\right] d s .
\end{aligned}
\end{aligned}
$$

Define the function $x(t)=x(t, a)$ by the formula

$$
\begin{aligned}
& x(t)= \begin{cases}w(t) & \text { if } t \bar{\epsilon}\left[\tau_{k} ; t_{k}\right], \\
z\left(t ; \tau_{k}, w\left(\tau_{k}+0\right)\right) & \text { if } t_{k}<t \leq \tau_{k}, \\
z\left(t ; \tau_{k}, w\left(\tau_{k}\right)\right) & \text { if } \tau_{k} \leq t<t_{k},\end{cases} \\
& x\left(t_{k}\right)=x\left(t_{k}-0\right) .
\end{aligned}
$$

From (55)-(57) it follows that the function $x(t)$ is differentiable for $t \neq \tau_{k}$ and satisfies the system

$$
\frac{d x}{d t}=g(x), t \neq \tau_{k}
$$

Moreover, 


$$
\Delta x\left(t_{k}\right)= \begin{cases}z\left(t_{k} ; \tau_{k}, w\left(\tau_{k}+0\right)\right)-w\left(t_{k}\right) & \text { if } t_{k}<\tau_{k}, \\ w\left(t_{k}\right)-z\left(t_{k} ; \tau_{k}, w\left(\tau_{k}\right)\right) & \text { if } t_{k}>\tau_{k}, \\ \Delta w\left(\tau_{k}\right) & \text { if } t_{k}=\tau_{k} .\end{cases}
$$

Let $t_{k}<\tau_{k}$. Then

$$
\begin{aligned}
\Delta x\left(t_{k}\right)= & z\left(t_{k} ; \tau_{k}, w\left(\tau_{k}+0\right)\right)-w\left(t_{k}\right) \\
= & \Delta w\left(\tau_{k}\right)+z\left(t_{k} ; \tau_{k}, w\left(\tau_{k}+0\right)\right)-w\left(\tau_{k}+0\right)+w\left(\tau_{k}\right)-w\left(t_{k}\right) \\
= & I\left(z\left(t_{k} ; \tau_{k}, w\left(\tau_{k}\right)\right)\right)+\int_{t_{k}}^{\tau_{k}}\left[g\left(z\left(s ; \tau_{k}, w\left(\tau_{k}+0\right)\right)\right)-g\left(z\left(s ; \tau_{k}, w\left(\tau_{k}\right)\right)\right)\right] d s \\
& +\int_{\tau_{k}}^{t_{k}} g\left(z\left(s ; \tau_{k}, w\left(\tau_{k}+0\right)\right)\right) d s-\int_{\tau_{k}}^{t_{k}} g\left(z\left(s ; \tau_{k}, w\left(\tau_{k}\right)\right)\right) d s \\
= & I\left(w\left(t_{k}\right)\right)=I\left(x\left(t_{k}\right)\right) .
\end{aligned}
$$

By analogous calculations it is proved that in the cases $t_{k}>\tau_{k}$ and $t_{k}=\tau_{k}$ the following equality holds as well:

$$
\Delta x\left(t_{k}\right)=I\left(x\left(t_{k}\right)\right) .
$$

Taking into account $z\left(t_{k}: \tau_{k}, w\left(\tau_{k}\right)\right)=x\left(t_{k}\right)$ and passing to the limit in the equality $\phi\left(z\left(t_{k}^{n} ; \tau_{k}, w_{n}\left(\tau_{k}\right)\right)\right)=0$, we get $\phi\left(x\left(t_{k}\right)\right)=0$, i.e. $t_{k}$ are moments of impulse effect for $x(t)$. Moreover, in view of condition A5, from (50) and (56) it follows that $x(t)$ has no other moments of impulse effect.

Hence the function $x(t)$ is a solution of system (1) with moments of impulse effect $t_{k}$. By (56), (50) and (51) the solution $x(t)$ and the moments $t_{k}$ satisfy (34) and (35).

We shall find a relation between the initial values $x^{0}=\operatorname{col}\left(x_{1}^{0}, \ldots, x_{n}^{0}\right)=x(0$, $a$ ) of the solutions $x(t, a)$ and the parameter $a \in D\left(\eta_{0}\right)$. Put $t=0$ into (56) and, in view of (52) and (30), obtain

$$
x^{0}=Y(0) a+\int_{0}^{\infty} G(0, s) f(s, a) d s+\sum_{k=1}^{\infty} G\left(0, \tau_{k}\right) b_{k}(a),
$$

where $f(t, a)$ and $b_{k}(a)$ are given by formulae (53) and (54).

From equality (32) it follows that

$$
Y(0) \operatorname{diag}\left(E_{1}, 0_{n-1}\right)=\operatorname{diag}\left(E_{1}, 0_{n-1}\right) .
$$

Let $c_{i j}$ and $C_{i j}(i, j=1, \ldots, n)$ be respectively the elements of the matrix $Y(0)$ and their cofactors. form

Taking into account (60) and (33), we conclude that equation (59) has the

$$
x_{1}^{0}=\sum_{j=2}^{n} c_{1 j} a_{j}+\xi\left(a_{2}, \ldots, a_{n}\right)
$$




$$
x_{i}^{0}=\sum_{j=2}^{n} c_{i j} a_{j} \quad(i=2, \ldots, n)
$$

where

$$
\xi\left(a_{2}, \ldots, a_{n}\right)=\left[\int_{0}^{\infty} G(0, s) f(s, a) d s+\sum_{k=1}^{\infty} G\left(0, \tau_{k}\right) b_{k}(a)\right]_{1}
$$

(Here $[x]_{1}$ denotes the first coordinate of the vector $x$ ).

Since the determinant $\Delta$ of system (62) is equal to

$$
\Delta=C_{11}=\operatorname{det} Y(0) \neq 0
$$

then system (62) is solvable with respect to $a_{2}, \ldots, a_{n}$, i.e.

$$
a_{j}=\sum_{k=2}^{n} d_{j k} x_{k}^{o} \quad(j=2, \ldots, n)
$$

where $d_{j k}$ are constants.

Substitute the result obtained into (61) and obtain

$$
x_{1}^{0}=\sum_{k=2}^{n} h_{k} x_{k}^{0}+\tilde{\xi}\left(x_{2}^{0}, \ldots, x_{n}^{0}\right),
$$

where $h_{k}$ are constants and $\tilde{\xi}\left(x_{2}^{0}, \ldots, x_{n}^{0}\right)=\xi\left(a_{2}, \ldots, a_{n}\right)$.

In view of (53), (54) and estimates (50), (51) we conclude that the function $\xi\left(a_{2}, \ldots, a_{n}\right)$ is differentiable in some neighbourhood of the point $0 \in D\left(\eta_{0}\right)$ and $\xi\left(a_{2}, \ldots, a_{n}\right)=o(|a|)$ as $|a| \rightarrow 0$.

Then, in virtue of $(63)$, the function $\tilde{\xi}\left(x_{2}^{0}, \ldots, x_{n}^{0}\right)$ is differentiable in some neighbourhood $\left(x_{2}^{0}\right)^{2}+\cdots+\left(x_{n}^{0}\right)^{2}<r^{2}$ and

$$
\tilde{\xi}\left(x_{2}^{0}, \ldots, x_{n}^{0}\right)=O(|x|) \quad \text { as }|x| \rightarrow 0 .
$$

This enables us to formulate the following assertions:

I. The graph of the function

$$
x_{1}^{0}=\sum_{k=2}^{n} h_{k} x_{k}^{0}+\tilde{\xi}\left(x_{2}^{0}, \ldots, x_{n}^{0}\right) \quad\left(\left(x_{2}^{0}\right)^{2}+\cdots+\left(x_{2}^{0}\right)^{2}<r^{2}\right)
$$

defines in $\mathbf{R}^{n}$ a smooth hypersurface $S$ and $0 \in S$.

II. The function

$$
\varphi(x)=x_{1}-\sum_{k=2}^{n} h_{k} x_{k}-\tilde{\xi}\left(x_{2}, \ldots, x_{n}\right)
$$

is differentiable in the neighbourhood $|x|<r$ and by (64), (65) and (30) we have

$$
\begin{aligned}
& \varphi(0)=0 \\
& \frac{\partial \varphi}{\partial x}(0)=\operatorname{col}\left(1,-h_{1}, \ldots,-h_{n}\right) \neq 0,
\end{aligned}
$$




$$
\frac{\partial \varphi}{\partial x}(0) g(p(0))=\frac{\partial \varphi}{\partial x}(0) \frac{d p}{d t}(0)=1 \neq 0
$$

III. For any $\rho \in(0, r)$ there exists $\rho_{1} \in(0, \rho)$ such that if $w \in \mathbb{R}^{n},|w|<\rho_{1}$, then the equation

$$
\varphi(z(t ; 0, w))=0
$$

has a unique solution $\hat{t}=\hat{t}(w) \in[-r, r]$ and

$$
|z(t ; 0, w)|<\rho \quad \text { for } t \in[0 ; \hat{t}] .
$$

Note that in the proof of Assertion III the arguments are as in the proof of Lemma 1.

Now let $\varepsilon>0$ and $\eta>0$ be given and $t_{0} \in \mathbb{R}_{+},\left|t_{0}-\tau_{k}\right|>\eta$.

Choose successively the numbers:

$\eta_{1} \in\left(0, \eta_{0}\right)$ so that $2 \tau K \eta_{1}<\eta_{0}$;

$\rho \in(0, \min (r, \varepsilon))$ so that if $\left(x_{2}^{0}\right)^{2}+\cdots+\left(x_{n}^{0}\right)^{2}<\rho^{2}$, then for the numbers $a_{j}$ defined by (63), we should have

$$
a_{2}^{2}+\cdots+a_{n}^{2}<\eta_{1}^{2}
$$

$\rho_{1} \in(0, \rho)$ so that Assertion III should hold.

Let $i T-T<t_{0}<i T$. Then $0<T_{0}=i T-t_{0}<T$ and

$$
p\left(t_{0}+T_{0}\right)=p(i T)=p(0)=0 .
$$

From Remark 3 and the continuous dependence of the solution of system (1) on the initial data it follows that there exists $\lambda>0$ such that for any $x_{0} \in \mathbb{R}^{n}$, $\left|x_{0}-p\left(t_{0}\right)\right|<\lambda$ we have

$$
\rho\left(x\left(t ; t_{0}, x_{0}\right), L\right)<\varepsilon \quad \text { for } t \in\left[t_{0}, t_{0}+T_{0}\right]
$$

and

$$
\left|x\left(t ; t_{0}, x_{0}\right)-p(t)\right|<\rho_{1}<\varepsilon \quad \text { for } t \in\left[t_{0}, t_{0}+T_{0}\right],\left|t-\tau_{k}\right|>\eta .
$$

In particular, for $w=x\left(t_{0}+T_{0} ; t_{0}, x_{0}\right)$, in view of (68) and (66), we have $|w|$ $<\rho_{1}$. Then by Assertion III there exists a unique $\hat{t} \in[-r, r]$ such that

$$
z(\hat{t} ; 0, w) \in S
$$

and

$$
|z(t ; 0, w)|<\rho \quad \text { for } t \in[0 ; \hat{t}] .
$$

Since system (1) is autonomous, then

$$
\begin{aligned}
x_{1} & =x\left(t_{0}+T_{0}+\hat{t} ; t_{0}, x_{0}\right)=x\left(t_{0}+T_{0}+\hat{t} ; t_{0}+T_{0}, x\left(t_{0}+T_{0} ; t_{0}, x_{0}\right)\right) \\
& =x\left(t_{0}+T_{0}+\hat{t} ; t_{0}+T_{0}, w\right)=x(\hat{t} ; 0, w)=z(\hat{t} ; 0, w) .
\end{aligned}
$$


Hence $x_{1} \in S$ and $\left|x_{1}\right|<\rho$ and to $x_{1}$ by (63) corresponds $a^{*} \in D\left(\eta_{1}\right)$. Then the solution $x\left(t ; 0, x_{1}\right)=x\left(t, a^{*}\right)$ of system (1) and its moments of impulse effect $t_{k}^{*}$ satisfy the estimates

$$
\begin{aligned}
& \left|t_{k}^{*}-\tau_{k}\right| \leq \delta_{k}=2 \tau K\left|a^{*}\right| e^{-\alpha \tau_{k}}<2 \tau K \eta_{1}<\eta \\
& \left|x\left(t ; 0, x_{1}\right)-p(t)\right|<2 K\left|a^{*}\right| e^{-\alpha t} \quad \text { for } t \geq 0,\left|t-\tau_{k}\right|>\delta_{k} .
\end{aligned}
$$

Having taken into account $p(t)=p\left(t+t_{0}+T_{0}\right)$,

$$
x\left(t ; 0, x_{1}\right)=x\left(t+t_{0}+T_{0}+\hat{t} ; t_{0}, x_{0}\right), \tau_{k}+t_{0}+T_{0}=\tau_{k+i q}
$$

and setting $s=t+t_{0}+T_{0}$, we obtain

$$
\left|x\left(s+\hat{t} ; t_{0}, x_{0}\right)-p(s)\right| \leq 2 K\left|a^{*}\right| e^{-\alpha\left(s-t_{0}-T_{0}\right)}
$$

for $s>t_{0}+T_{0},\left|s-\tau_{k+i q}\right|>2 \tau K\left|a^{*}\right| e^{-\alpha \tau_{k}}$.

For $s \in\left[\tau_{k+i q}-\delta_{k}, \tau_{k+i q}+\delta_{k}\right]$ there holds either the estimate

$\left|x\left(s+\hat{t} ; t_{0}, \quad x_{0}\right)-p\left(\tau_{k+i q}-\delta_{k}\right)\right| \leq 2 K\left|a^{*}\right|\left((1+\tau d) e^{2 \alpha \eta}+\tau d e^{\alpha \eta}\right) e^{-\alpha\left(s-t_{0}-T_{0}\right)}$

or the estimate

$$
\left|x\left(s+\hat{t} ; t_{0}, t_{0}\right)-p\left(\tau_{k+i q}+\delta_{k}\right)\right| \leq 2 K\left|a^{*}\right|\left(1+\tau d+\tau d e^{\alpha \eta}\right) e^{-\alpha\left(s-t_{0}-T_{0}\right)},
$$

where $d=\sup _{x \in \bar{D}}|g(x)|$.

Then from (70) it follows that the solution $p(t)$ has the property asymptotic phase, from (70)- (72) it follows that $p(t)$ is orbitally attractive and (69)-(72) and (67) imply that $p(t)$ is orbitally stable, i.e. it is orbitally asymptotically stable.

Remark 4. Let the manifold $M$ divide the domain $\Omega$ into $q$ disjoint parts:

$$
\begin{gathered}
\Omega=\Omega_{1} \cup \cdots \cup \Omega_{q} \cup M, \\
\Omega_{i} \cap \Omega_{j}=\varnothing, \Omega_{i} \cap M=\varnothing, i, j=1, \ldots, q, i \neq j
\end{gathered}
$$

and

$$
\Gamma_{k} \subset \Omega_{k} \cup M, k=1, \ldots, q .
$$

Let the function $g_{k}: \Omega \rightarrow \mathbb{R}^{n}, k=1, \ldots, q$ be differentiable in $D_{k}(H)$ and continuous in $\bar{D}_{k}(H)$.

Then Theorem 1 holds if

$$
g(x)=g_{k}(x) \quad \text { for } x \in \Omega_{k} \cap \bar{D}_{k}(H)
$$

or if condition A5 is replaced by the following condition $\mathrm{A5}^{\prime}$. For any $h \in(0, H)$ there exists $\gamma>0$ such that:

$$
\rho(x, M) \geq \gamma \text { for } k=1, \ldots, q \text { and } x \in \Gamma_{k} \backslash\left(\bar{B}_{h}\left(p\left(\tau_{k}\right)\right) \cup \bar{B}_{h}\left(p\left(\tau_{k-1}+0\right)\right)\right) .
$$


$x+I(x) \in \Omega_{k} \cup M$ for $x \in M \cap B_{h}\left(p\left(\tau_{k-1}\right)\right)$.

$\frac{\partial \phi}{\partial x}\left(p\left(\tau_{k-1}+0\right)\right) g\left(p\left(\tau_{k-1}+0\right)\right) \neq 0, k=1, \ldots, q$.

Consider the case $n=2$. Assume that system (1) has a T-periodic solution $p(t)\left(\frac{d p}{d t} \neq 0\right)$ and in the interval $(0, T]$ the solution $p(t)$ has $q$ moments of impulse effect. Let $Y(t)$ be the normalized for $t=0$ fundamental matrix of the system in variations (4).

Then the multiplicators $\rho_{1}, \rho_{2}$ of system (4) satisfy the equation

$$
\operatorname{det}\left(Y(T)-\rho E_{2}\right)=0
$$

or

$$
\rho^{2}-\rho \operatorname{Tr} Y(T)+\operatorname{det} Y(T)=0
$$

Since system (4) has a non-trivial T-periodic solution $\frac{d p}{d t}$, then one of its multiplicators is $\rho_{1}=1$. From (73) and Viète's formulae it follows that

$$
\rho_{2}=\operatorname{det} Y(T)
$$

or, in detail,

$$
\rho_{2}=\prod_{k=1}^{q} \operatorname{det}\left(E_{2}+N_{k}\right) e \int_{0}^{T} \operatorname{Tr} \frac{\partial g}{\partial x}(p(t)) d t
$$

Let system (1), in scalar notation, have the form

$$
\begin{aligned}
& \mid \begin{array}{l}
\frac{d x}{d t}=P(x, y) \\
\frac{d y}{d t}=Q(x, y) \quad \text { if }(x, y) \bar{\in} M,
\end{array} \\
& \mid \begin{array}{l}
\Delta x=a(x, y) \\
\Delta y=b(x, y) \quad \text { if }(x, y) \in M,
\end{array}
\end{aligned}
$$

the set $M$ is defined by the equation

$$
\phi(x, y)=0
$$

and $p(t)=\operatorname{col}(\xi(t), \eta(t)),\left|\frac{d \xi}{d t}(t)\right|+\left|\frac{d \eta}{d t}(t)\right| \neq 0$.

Then system (4) assumes the form 


$$
\begin{aligned}
& \mid \begin{array}{l}
\frac{d u}{d t}=\frac{\partial P}{\partial x}(\xi(t), \eta(t)) u+\frac{\partial P}{\partial y}(\xi(t)) v, t \neq \tau_{k}, \\
\frac{d v}{d t}=\frac{\partial Q}{\partial x}(\xi(t), \eta(t)) u+\frac{\partial Q}{\partial y}(\xi(t), \eta(t), \eta(t)) v, t \neq \tau_{k},
\end{array} \\
& \mid \begin{array}{ll}
\left.\Delta u\right|_{t=\tau_{k}}=A_{k} u+B_{k} v, & k=1,2, \ldots, \\
\left.\Delta v\right|_{t=\tau_{k}}=C_{k} u+D_{k} v, & k=1,2, \ldots,
\end{array}
\end{aligned}
$$

where the constants $A_{k}, B_{k}, C_{k}$ and $D_{k}$ have been calculated according to formula (5).

Then from (74) and (76) we obtain

$$
\rho_{2}=\prod_{k=1}^{q} \Delta_{k} e \int_{0}^{T}\left[\frac{\partial P}{\partial x}(\xi(t), \eta(t))+\frac{\partial Q}{\partial y}(\xi(t), \eta(t))\right] d t
$$

where $\Delta_{k}=\operatorname{det}\left(E_{2}+N_{k}\right)=1+A_{k}+D_{k}+A_{k} D_{k}-B_{k} C_{k}$.

After detailed calculations we find that

$$
\Delta_{k}=\frac{P_{+}\left(\frac{\partial b}{\partial y} \frac{\partial \phi}{\partial x}-\frac{\partial b}{\partial x} \frac{\partial \phi}{\partial y}+\frac{\partial \phi}{\partial x}\right)+Q_{+}\left(\frac{\partial a}{\partial x} \frac{\partial \phi}{\partial y}-\frac{\partial a}{\partial y} \frac{\partial \phi}{\partial x}+\frac{\partial \phi}{\partial y}\right)}{P \frac{\partial \phi}{\partial x}+Q \frac{\partial \phi}{\partial y}}
$$

where $P, Q, \frac{\partial a}{\partial x}, \frac{\partial a}{\partial y}, \frac{\partial b}{\partial x}, \frac{\partial b}{\partial y}, \frac{\partial \phi}{\partial x}, \frac{\partial \phi}{\partial y}$ have been calculated at the point $\left(\xi\left(\tau_{k}\right), \eta\left(\tau_{k}\right)\right)$ and $P_{+}=P\left(\xi\left(\tau_{k}+0\right), \eta\left(\tau_{k}+0\right)\right)$,

$$
Q_{+}=Q\left(\xi\left(\tau_{k}+0\right), \eta\left(\tau_{k}+0\right)\right) .
$$

Thus for $n=2$ the following corollary holds.

\section{Corollary 2 (Analogue of Poincaré's criterion).}

The solution $p(t)=\operatorname{col}(\xi(t), \eta(t))$ of system (75) is orbitally asymptotically stable and has the property asymptotic phase if the multiplicator $\rho_{2}$ calculated by formula (77) satisfies the condition $\left|\rho_{2}\right|<1$.

Example 1. Consider the second order equation

$$
\ddot{x}+2 h \dot{x}+\omega^{2} x=0 \quad(0<h<\omega) .
$$

Let the solution $x(t)$ be subject to impulse effect at the moment $t=\tau_{k}$ :

$$
x\left(\tau_{k}+0\right)=x\left(\tau_{k}\right), \dot{x}\left(\tau_{k}+0\right)=\dot{x}\left(\tau_{k}\right)+b\left(x\left(\tau_{k}\right), \dot{x}\left(\tau_{k}\right)\right),
$$

where $\tau_{k}$ is a moment of impulse effect if

$$
x\left(\tau_{k}\right)=0, \dot{x}\left(\tau_{k}\right) \geq 0 .
$$

The equation with impulse effect $(79)-(81)$ represents the simplest 
mathematical model of a clock-work [12].

Set $\dot{x}=y$ and write down equations (79)-(81) in the form of a system:

$$
\begin{array}{ll}
\dot{x}=y & \\
\dot{y}=-\omega^{2} \times-2 h y & \text { if }(x, y) \bar{\in} M \\
\Delta x=0 & \\
\Delta y=b(x, y) & \text { if }(x, y) \in M,
\end{array}
$$

where $M$ is defined by the conditions

$$
\phi(x, y) \equiv x=0, y \geq 0 .
$$

System (82)-(83) has a non-trivial $T$-periodic solution $x=\xi(t), y=\eta(t)$ which coincides with the $T$-periodic continuation of the solution

$$
\begin{aligned}
& x=\frac{y_{0}}{\beta} e^{-h t} \sin \beta t, \\
& y=y_{0} e^{-h t}\left(\cos \beta t-\frac{h}{\beta} \sin \beta t\right)
\end{aligned}
$$

of (82) for $t \in(0, T]$, where $y_{0}$ is chosen so that

$$
y_{0} e^{-\boldsymbol{h} T}+b(x(T), y(T))=y_{0}
$$

and

$$
T=2 \pi \beta^{-1}, \beta=\sqrt{\omega^{2}-h^{2}} .
$$

In our case $q=1, \tau_{1}=T, x(T)=0, x(T+0)=0$,

$$
\begin{aligned}
& y(T)=y_{0} e^{-h T}, y(T+0)=y_{0} e^{-h T}+b(x(T), y(T))=y_{0}, \\
& \frac{\partial a}{\partial x}=\frac{\partial a}{\partial y}=\frac{\partial \phi}{\partial y}=0, \quad \frac{\partial \phi}{\partial x}=1, \quad \frac{\partial P}{\partial x}=0, \quad \frac{\partial Q}{\partial y}=-2 h, \\
& \left.\int_{0}^{T}\left[\frac{\partial P}{\partial x}(\xi(t)), \eta(t)\right)+\frac{\partial Q}{\partial y}(\xi(t), \eta(t))\right] d t=-2 h T . \\
& \text { i) Let } b(x, y)=p>0 .
\end{aligned}
$$

Then $\frac{\partial b}{\partial x}=\frac{\partial b}{\partial y}=0$,

$$
\begin{aligned}
& \Delta_{1}=\frac{P_{+}}{P}=\frac{y(T+0)}{y(T)}=\frac{y_{0}}{y_{0} e^{-h T}}=e^{h T}, \\
& \rho_{2}=e^{h T} e^{-2 h T}=e^{-h T} .
\end{aligned}
$$

ii) Let $b(x, y)=\sqrt{y^{2}+\varepsilon}-y \quad(\varepsilon>0)$. 
Then

$$
y_{0} e^{-h T}+\sqrt{y_{0}^{2} e^{-2 h T}+\varepsilon}-y_{0} e^{-h T}=y_{0}, \frac{\partial b}{\partial x}=0, \frac{\partial b}{\partial y}=\frac{y}{\sqrt{y^{2}+\varepsilon}}-1
$$

and

$$
\Delta_{1}=\frac{P_{+}\left(\frac{\partial b}{\partial y}+1\right)}{P}=\frac{y(T+0)}{y(T)} \cdot \frac{y(T)}{\sqrt{y^{2}(T)+\varepsilon}}=\frac{Y_{0}}{\sqrt{y_{0}^{2} e^{-2 h T}+\varepsilon}}=1 .
$$

Hence $\rho_{2}=e^{-2 h T}$.

In the case (i) and (ii) $\rho_{2} \in(0,1)$. Hence, by Corollary 2, the solution $x$ $=\xi(t), y=\eta(t)$ of the system (82) is orbitally asymptotically stable. It is to this solution that the normal work of the clock corresponds.

Example 2. The linear system with impulse effect

$$
\begin{aligned}
& \frac{d x}{d t}=\left\{\begin{array}{cc}
\frac{k r y}{k r S-1}, & \text { if }|x|<\frac{V_{s}}{2} \\
-k r y, & \text { if }|x| \geq \frac{V_{s}}{2}
\end{array}\right. \\
& \frac{d y}{d t}= \begin{cases}\frac{x}{k r L C}+\frac{r y}{L(k r S-1)}, & \text { if }|x|<\frac{V_{s}}{2} \\
\frac{x}{k r L C}-\frac{r y}{L}, & \text { if }|x| \geq \frac{V_{s}}{2}\end{cases} \\
& \Delta x=-2 S k r x, \Delta y=-\frac{2 S r}{L} x, \text { if }|x|=\frac{V_{s}}{2},
\end{aligned}
$$

models the work of the electronic scheme given on (Fig. 1) ([13], ch. V. § 17).

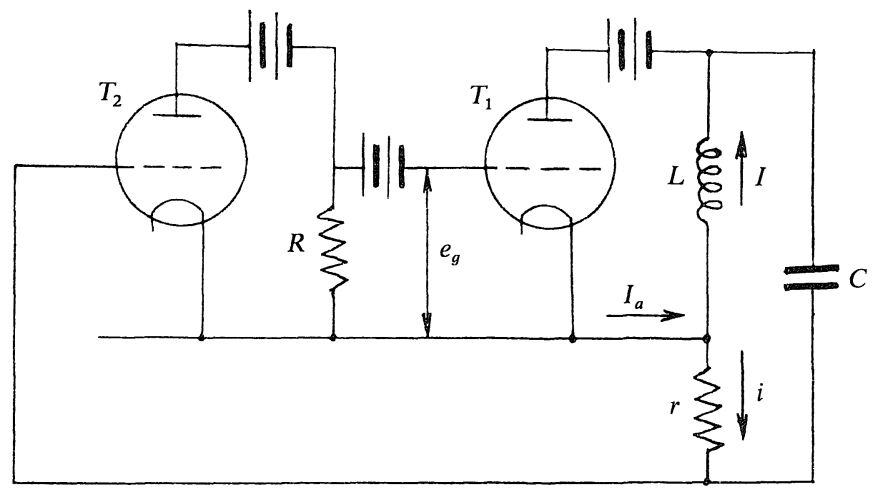

Fig.1 
Here $k$ is the amplification factor which depends on the parameters of the tube $T_{2}$ and on the anode resistance $R, x=e_{g}=k r i$ is the lattice tension of the tube $T_{1} ; y=\frac{d I}{d t} ; S=\frac{I_{s}}{V_{s}}$ is the obliquity of the lattice characteristic of the tube $T_{1}$; $I_{s}$ and $V_{s}$ are respectively the current intensity and tension of satiation of the tube $T_{1} ; k r S-1>0$.

In $[13]$ it is proved that in the cases when $L$ is small $\left(L \ll \frac{C r^{2}}{4}\right)$ or $L$ is large $\left(L \gg \frac{C r^{2}}{4}\right)$ system $(84)$ has a unique non-zero periodic solution $p(t)$ and the period $T$ of this solution is estimated:

$$
\begin{aligned}
& T \approx \frac{\pi}{\sqrt{\frac{1}{L C}+\frac{r^{2}}{4 L^{2}}}} \quad\left(\text { for } L \gg \frac{C r^{2}}{4}\right) \\
& T \approx 2 \frac{L}{r} \ln (2 S k r-1) \quad\left(\text { for } L \ll \frac{C r^{2}}{4}\right)
\end{aligned}
$$

The phase trajectory of $p(t)$ is given on Fig. $2\left(\right.$ for $\left.L \gg \frac{C r^{2}}{4}\right)$ and on Fig. 3 (for $\left.L \ll \frac{C r^{2}}{4}\right)$.

The motion of the mapping point $(x(t), y(t))$ is realized in the set defined by the inequality $|x| \geq \frac{V_{s}}{2}$. This motion is continuous from point $A_{2}$ to point $A_{3}$ and from point $A_{4}$ to point $A_{1}$ and by jumps from point $A_{1}$ into point $A_{2}$ and from point $A_{3}$ into point $A_{4}$ (Fig. 2, Fig. 3).

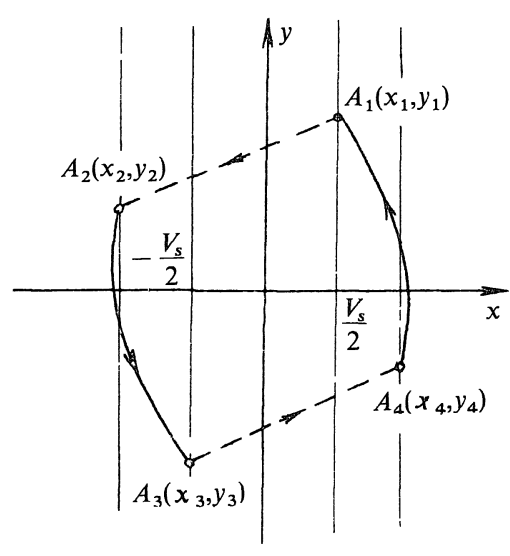

Fig.2

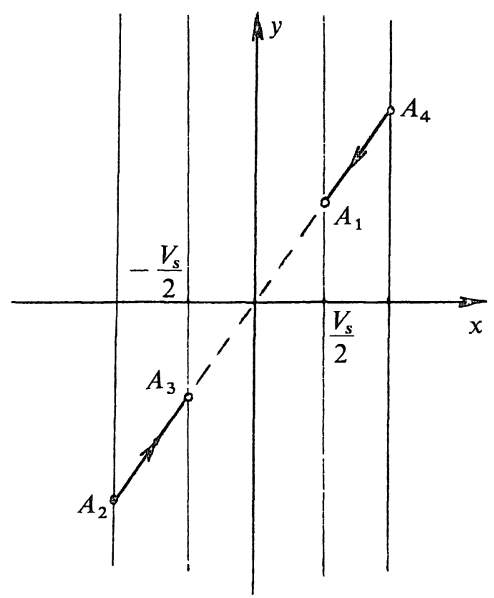

Fig.3 
Using the above notations we obtain

$$
\begin{aligned}
& P(x, y)=-k r y, Q(x, y)=\frac{x}{k r L C}-\frac{r y}{L}, \\
& a(x, y)=-2 S k r x, b(x, y)=-\frac{2 S r}{L} x, \phi(x, y)=|x|-\frac{V_{s}}{2}, \\
& \frac{\partial P}{\partial x}=0, \frac{\partial P}{\partial y}=-k r, \frac{\partial Q}{\partial x}=\frac{1}{k r L C}, \frac{\partial Q}{\partial y}=-\frac{r}{L}, \\
& \frac{\partial a}{\partial x}=-2 S k r, \frac{\partial a}{\partial y}=0, \frac{\partial b}{\partial x}=-\frac{2 S r}{L}, \frac{\partial b}{\partial y}=0 \\
& \frac{\partial \phi}{\partial x}\left(\frac{V_{s}}{2}, y\right)=1, \frac{\partial \phi}{\partial x}\left(-\frac{V_{s}}{2}, y\right)=-1, \frac{\partial \phi}{\partial y}=0 .
\end{aligned}
$$

Then

$$
\begin{aligned}
& \int_{0}^{T}\left(\frac{\partial P}{\partial x}+\frac{\partial Q}{\partial y}\right) d t=-\frac{r T}{L} \\
& \Delta_{1}=\frac{P_{+}}{P}=\frac{P\left(x_{2}, y_{2}\right)}{P\left(x_{1}, y_{1}\right)}=\frac{y_{1}-\frac{2 S r}{L} x_{1}}{y_{1}}=\frac{y_{1}-\frac{r}{L} I_{s}}{y_{1}}, \\
& \Delta_{2}=\frac{P+}{P}=\frac{P\left(x_{4}, y_{4}\right)}{P\left(x_{3}, y_{3}\right)}=\frac{-y_{1}-\frac{2 S r}{L} x_{1}}{y_{1}}=\Delta_{1}, \\
& \rho_{2}=\left(\frac{y_{1}-\frac{r}{L} I_{s}}{y_{1}}\right)^{2} e^{-\frac{r T}{2}} .
\end{aligned}
$$

In the cases when $L \gg \frac{C r^{2}}{4}$ or $L \ll \frac{C r^{2}}{4}$, the condition $0<\rho_{2}<1$ is satisfied. Then the periodic solution of system (84) is orbitally asymptotically stable.

\section{References}

[1] Mil'man, V. D. and Myshkis, A. D., On the stability of motion in the presence of impulses, Siberian Math. J., 1 (1960), 233-237 (in Russian).

[2] Myshkis, A. D. and Samoilenko, A. M., Systems with impulses in prescribed moments of the time, Math. Sb., 74 (1967), 202-208 (in Russian)

[3] Samoilenko, A. M. and Perestiuk, N. A., Stability of the solutions of differential equations with impulse effect, Differencialnye Uravn. 11 (1977), 1981-1992 (in Russian).

[4] - On the stability of the solutions of systems with impulse effect, Differencialnye Uravn. 11 (1981), 1995-2001 (in Russian). 
[ 5] Simeonow, P. S. and Bainov, D. D., Asymptotic equivalence of two systems of differential equations with impulse effect, Systems \& Control Letters 3 (1983), 297-301.

[6] - Stability under persistent disturbances for systems with impulse effect, Journal of Math. An. and Appl. 109 (1985), 546-563.

[7] — The second method of Liapunov for systems with an impulse effect, Tamkang Journal of Mathematics, 16 (1985), 19-40.

[8] - Stability of the solutions of singularly perturbed systems with impulse effect, COMPEL. 5 (1986), 95-108.

[9] — - Stability with respect to part of the variables in systems with impulse effect, Journal of Math. An. and Appl., 117 (1986), 247-263.

[10] Andronov, A. A. and Vitt, A. A., On the stability by Liapunov, Journ. of Exp. and Theor. Physics, 3 (1933) (in Russian).

[11] Demidovich, B. P., Lectures on the Mathematical Theory of Stability, Nauka, Moscow, 1967. (in Russian).

[12] Butenin, N. V., and Neimark, Yu. I. Fufaev, N. A., Introduction to the Theory of Nonlinear Oscillations, Nauka, Moscow, 1976 (in Russian).

[13] Andronov, A. A., Vitt, A. A. and Khaĭkin, S. E., Oscillation Theory, Nauka, Moscow, 1981 (in Russian). 